\title{
Testing using Telecommunications Management
}

\author{
Ronald C. van Wuijtswinkel and Marc F. Witteman
}

PTT Research, P.O. Box 421, 2260 AK Leidschendam, the Netherlands

The conventional protocol conformance test methods are not able to test all system components effectively. If these problems are to be overcome, direct access to the protocols is absolutely vital. The ISO 9646 conceptual test method offers direct access to the Implementation Under Test. However, this method requires an extra test interface. A logical step would be the use of the OSI management interface to perform protocol conformance tests. This paper describes the technical details and opportunities of a new ISO standard that is currently being developed. With this new approach, the test process becomes cheaper and faster, and test coverage will be much higher.

\section{Keyword Codes C.2.2}

Keywords: Computer Communication Networks, Network Protocol, Telecommunications Management

\section{INTRODUCTION}

The ISO-9646 'remote test method' [1], although frequently used in the field of conformance testing, is not very powerful, because it has no direct access to the IUT. Each IUT is surrounded by other protocols that prevent full control and cbservation of the IUT. The shortcomings of the remote test method lead to the following problems:

- Testing is expensive, because complex test equipment is needed;

- Testing is time-consuming in relation to the time required for specification and implementation;

- Testing does not create real confidence in the SUT, because the test coverage is low.

To solve these problems, direct access to the separate protocol (sub)layers is absolutely vital. Only by direct access cheaper generic test systems can be created and a shorter testing phase can be achieved. ISO 9646 defines the 'conceptual' test method (see figure 1). In the conceptual test method, the protocol layer can be controlled and observed at its service boundaries, where communication between adjacent the protocol layers takes place. The access points on the protocol boundaries are called the Points of Control and Observation (PCO).

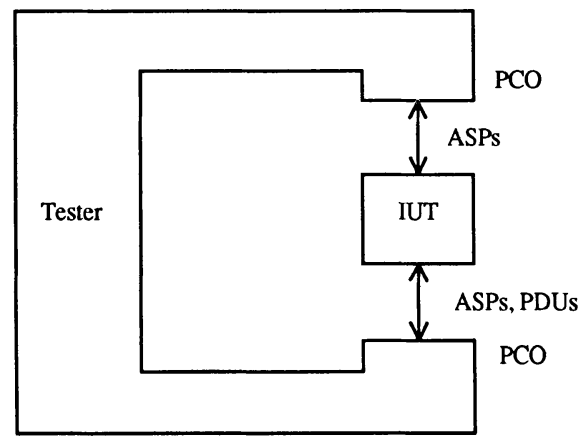

Figure 1. The ISO-9646 conceptual test method. 
The concept of direct access, using the conceptual test method has already been studied in a scientific context. Zeng introduced the Ferry principle [2] as a solution to the test access problem. This principle has been improved and extended until it proved fit for practical use. Within the European RACE program the Ferry principle is currently applied for testing experimental ATM broadband systems [3].

Apart from the significant increase in testability the ferry systems have the disadvantage to require an external interface solely for the purpose of testing. This is a major drawback that might prevent a wider application of the promises of the conceptual test method as used in the Ferry principle. Therefore there appears to be a need for re-using existing standard interfaces for the purpose of testing protocol layers.

Most large telecommunications systems have an external interface for management purposes. In the future the management interfaces of many large telecommunications systems will be based on OSI management. A logical step was the choice to use the management interface to perform protocol conformance tests. Possible advantages in addition to the ones from the Ferry principle can be summarised as follows:

- The telecommunication management standard already supports some form of testing;

- Facilities used for managing purposes can be reused for testing purposes;

- In todays test environments management control is already being used, e.g. to configure a SUT for testing purposes;

- No extra constraints on an implementation regarding an external test interface;

The following sections describe what Telecommunications Management is and how it can be used for testing.

\section{OSI MANAGEMENT}

Many manufacturers of telecommunications management systems presently use their own nonstandardised management interfaces. However, future management systems are likely to be based on standardised concepts; ITU-TS (formerly CCITT) is developing the Telecommunications Management Network (TMN) concept. The TMN is based on a layered management architecture consisting mainly of management systems that manage the network elements. Information exchange between them takes place via standardised interfaces. For these interfaces, the OSI management concept is used, i.e. OSI Management standardises the way in which management information can be exchanged between open systems [4], [5]. In the concept of TMN open systems can either consists of managing systems or managed systems.

\subsection{Fault management}

OSI Management subdivides the management concept into 5 general areas:

- Accounting management;

- Configuration management;

- Fault management;

- Performance management;

- Security management.

Fault management is defined in [4] to encompass fault detection, isolation and the correction of abnormal operation of the OSI environment. One area of the OSI fault management is called diagnostic testing. This has been standardised in the so-called Test management function, that is discussed in the next section. 


\subsection{Test Management Function}

To standardise the remote control of tests, the Test Management Function [7] is defined. Among other things, a test model has been defined that is based on the manager-agent model [6]. In this so called manager-agent test model, the Operations System (Managing System) controls a test using a test conductor and the Network Element (Managed System) carries out the test using a test performer (see figure 2). Upon execution of a test, the test conductor directs a test request to the test performer. The test request indicates which managed object refers to the functionalities that have to be tested. This managed object is called a Managed Object Referring to Test (MORT). Also Associated Objects (AO) are defined. AOs represent managed objects that are also involved in the tests.

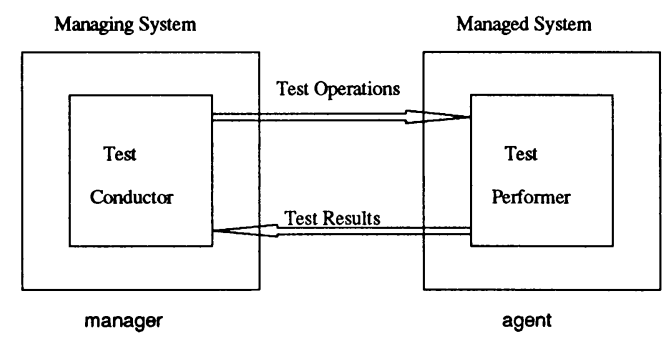

Figure 2. The manager - agent test model.

\subsection{Test Categories}

The generic Test Management Function applies to many kinds of tests. In a separate OSI Systems Management Function, named the Confidence and Diagnostic Test Categories [8], various test categories are defined. A Test Category specifies test characteristics and test management information for specific kinds of tests. One of them, the Resource Boundary Test Category (RBTC), is recently added to the existing test categories to allow direct access tests.

\subsection{Resource Boundary Test Category}

\subsubsection{Purpose of test category}

A system may consist of many resources. The purpose of the Resource Boundary Test Category is to verify the correctness of separate resources internal to the system. The behaviour of the resource is tested by controlling and observing the interactions between the resource and its environment. Points of Control and Observation (PCOs) will be located at the boundaries of the resource. At these points the information exchanged between the resources under test is defined in terms of signals. The behaviour of the resource is tested by inserting and observing test signals at the PCOs. Then it can be verified whether the signals generated by the resource conform to the behaviour specification of the resource. This verification however, does not take place within the System Under Test, but is actually performed by the Managing System that contains the Test Conductor.

\subsubsection{MORT requirements and Associated object requirements}

The MORTs represent the resources that are being tested by the resource boundary test. A MORT is an identifiable resource. The test can be applied to one or more adjacent resources. A resource can however, recursively contain several resources. When several adjacent resources are tested in one test, it is called multi-resource testing. In the case of multi-resource testing, each resource that 
is tested is represented by a MORT as shown in figure 3. Figure 4 shows the model for Resource Boundary Testing.

As PCOs are located at the boundaries of the resources, each of them may have connections with several resources. A PCO is represented by an AO. Apart from observing and inserting signals the PCO can switch the information stream between the adjacent resources on or off. In this way one or several resources can be isolated from the system, and tested without disturbing the systems internal state.

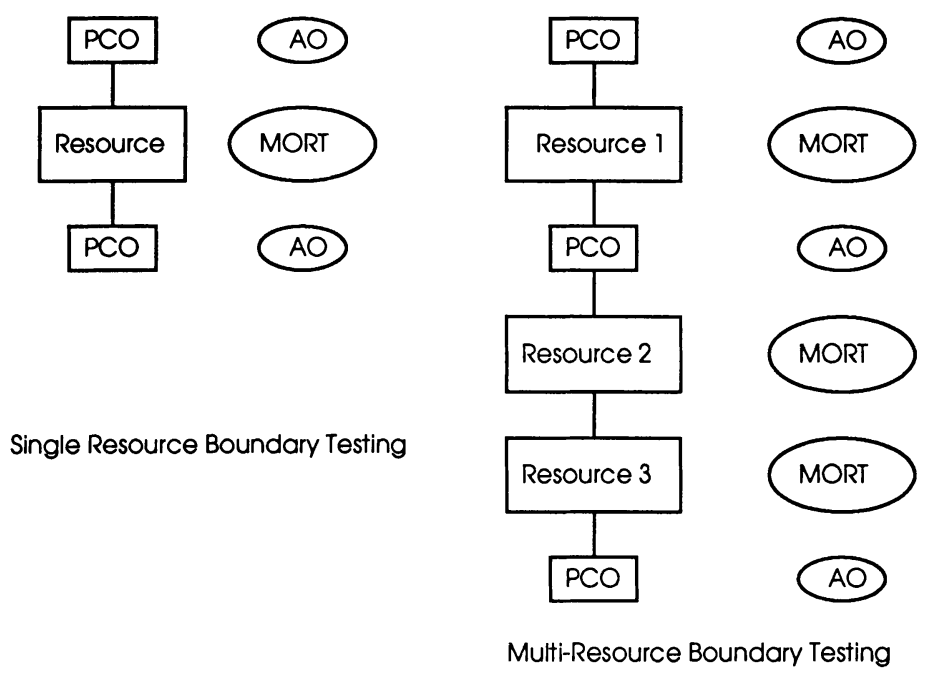

Figure 3: Possible configurations of MORTs and AOs for Resource Boundary Testing

A resource boundary test session is started by means of a test request, that specifies the MORTs and the AOs. The test session is terminated by means of a test termination. In between, several tests can be executed. Each test can consist of several test events. Test events are representations of signals that have to be inserted or to be received at a PCO.

The first series of test events that have to be executed can be specified in the test request (when the Test Object is created). Afterwards, the test events to be executed can repeatedly be specified by configuration of the Test Object using the PT-SET service, see section 9.3 of ITU-T Rec. X.745 I ISO/IEC 10164-12, the Test Management function [7].

The event list, included in the test request or PT-SET service request, may contain one or more test events. In the case of non-deterministic behaviour, the sequence of test events will generally contain a single signal that has to be inserted. An expected response is then not specified. Any signal that is received in this case shall immediately be forwarded to the managing system. The managing system is responsible for the validation of these signals.

Several signals could be listed in the sequence of events when completely determined behaviour of the resource under test is expected. In that case also expected responses in terms of signals are 
specified. The sequence of events consists then of both send and receive signals. This option enables a faster test execution, and is very suitable when time critical tests shall be performed.

Managing System

Managed System

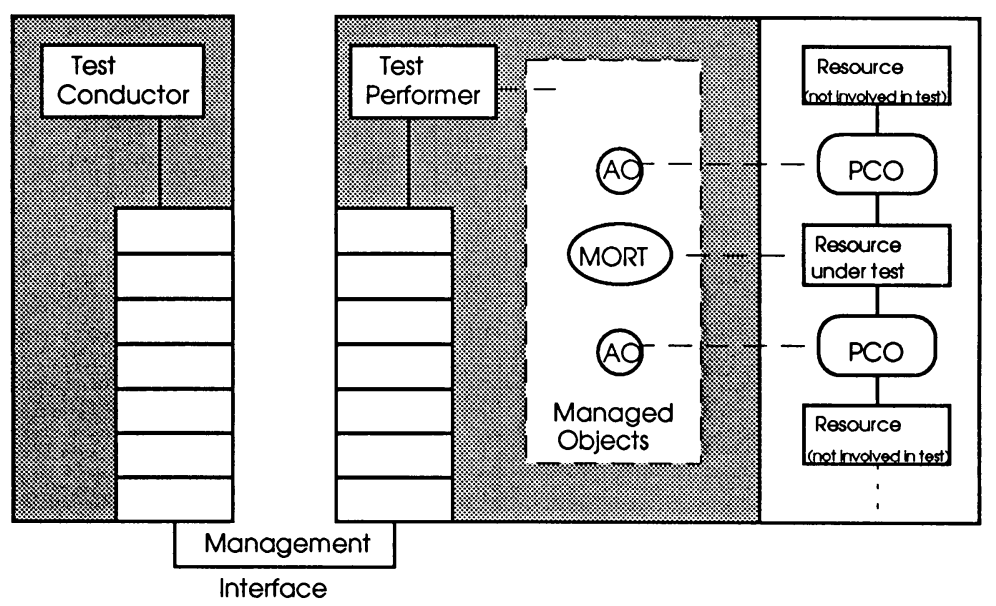

Figure 4: Test model for Resource Boundary Testing

At one PCO, signals may be received from, as well as inserted in, any of the connected resources. As the received signals may deviate from the expected results, the test course (the interactions between managing system and managed system) is not pre-defined. The test course depends on the actions of both the managing system and the tested resource.

The test performer shall consider the list containing the sequence of events to be active as soon as it starts handling the events. The sequence of events will be handled sequentially while this list is active. The list will remain active until all events are successfully handled or when a failure has occurred.

The handling of events differs for send and receive events. In case of a send event the test performer shall first await the expiration of an optional timer related to the event. Then it shall insert the signal at the given PCO. In case of a receive event, the test performer shall wait for the receipt of the indicated signal before continuing with the next event. The receipt of another signal then the next one in the list, or the expiration of a wait duration timer related to a receive signal causes a failure. In that case the handling of the list is terminated.

\subsubsection{Initiation specific to test category}

To start a test session the managing system shall issue a test request. In this test request all involved AOs are indicated. These AOs represent the PCOs that are involved during the test session. Additionally the state of each AO will be included. This is used to configure the PCOs in the appropriate way for the test session.

Furthermore the test request may contain a list of events. These lists can however be provided by additional PT-SET requests as well. An event list will contain at least the following information for each event: 
- $\quad$ Signal type;

- Parameters of the signal and their values;

- Direction of the signal (inserted or received);

- Identity of the MORT into which the signal is inserted or from which it is received;

- Identity of the AO where the signal is inserted or received;

- Wait duration timer value

\subsubsection{Reporting and termination events}

The managed system may spontaneously report intermediate results under a number of circumstances:

1. Completion of last signal in sequence of test events;

2. Receipt of a signal at a PCO while no event sequence list is active and therefore no receive signal is to be matched;

3. Receipt of a signal at a PCO while another receive signal is to be matched according to the active event sequence list;

4. Wait duration timer expired in the case of a receive signal;

There is one event that triggers the termination of the Resource Boundary test and the reporting of the final test results:

- Receipt of termination request, sent by the test conductor

The report may contain, depending on the reason for this report, the following information:

- Intermediate Resource Boundary Test outcome;

- $\quad$ Signal Type of the received signal;

- $\quad$ Parameters of the signal and their values;

- Identity of the MORT from which the signal was received; and

- Identity of the AO where the signal was received

\subsubsection{Test Termination}

The test can only be terminated by the managing system. This is due to the fact that all intelligence with respect to the tests is contained within the managing system. Therefore, the managing system should also decide when a test is terminated.

\subsubsection{Example application of the resource boundary test category}

The following example will show how the mechanism provided by the Resource Boundary Test Category can be applied in practical test execution.

Suppose we are testing a protocol X using an upper tester UT_X and a lower tester LT_X.We would like to test whether the protocol is capable of sending signal $C$ to the lower tester after the receipt of signals A and B from the upper tester. A TTCN-like description of this case would be the following:

\begin{tabular}{|l|c|l|}
\hline 1 & UT_X $\quad$ A & \\
\hline 2 & UT_X ! & \\
\hline 3 & LT_X ? C & Verdict PASS \\
\hline
\end{tabular}

In case of success this scenario might result in the exchange of test management messages shown in figure 5 . 
Test Conductor

Test Performer

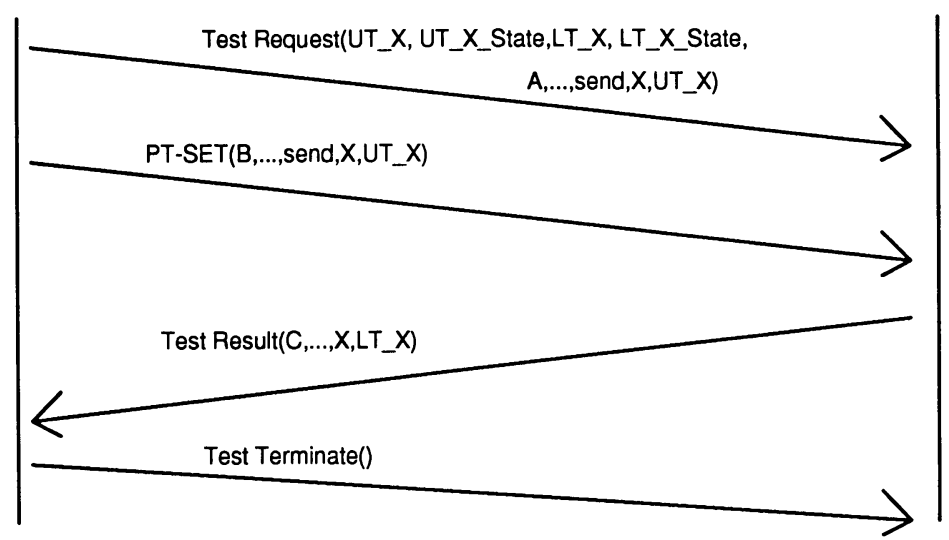

Figure 5. Example of test management message flow

The test conductor starts the test session by sending a test request indicating the PCOs (UT_X, LT_X) involved and their respective states. Furthermore it already contains a first event: send message A to resource X from PCO UT_X. Immediately hereafter the test conductor issues a PTSET operation to request the sending of event $B$ to resource $X$ from PCO UT_X. Then the test conductor awaits the response of the test performer. The test result contains the indication that signal C is received from resource X at PCO LT_X. Finally the test conductor ends the test session by sending a Test Terminate.

\section{TESTING USING OSI MANAGEMENT}

As described in section 2.3 the Test Management Function and especially the Resource Boundary Test Category can be used for different kinds of tests. Practical applications may be related to interoperability (inter working) testing, distributed system (network) testing, testing in the operational phase, prototype testing and hardware testing. In this section examples are given to use OSI management for protocol conformance testing and interoperability testing.

\subsection{Conformance Testing}

The OSI Test management function can be used to facilitate protocol conformance testing as shown in figure 6. With such an approach, exact coding for the ASP implementations would no longer need to be specified, because they could be mapped on managed objects.

\subsection{Interoperability Testing}

Figure 7 shows an example of a practical implementation of interoperability testing using OSI Management. A tester operates the test conductor process in a managing system. The managing system is connected to both managed system A and managed system B. In both managed systems a test performer process is running and managed objects are visible, that represent the protocol layers and PCOs. In the example, the communication between the lower four protocol layers of managed systems A and B are tested. The tester determines which protocol messages need to be inserted in the protocol layers via the PCOs and obtains the results. 
Test System

Network Element

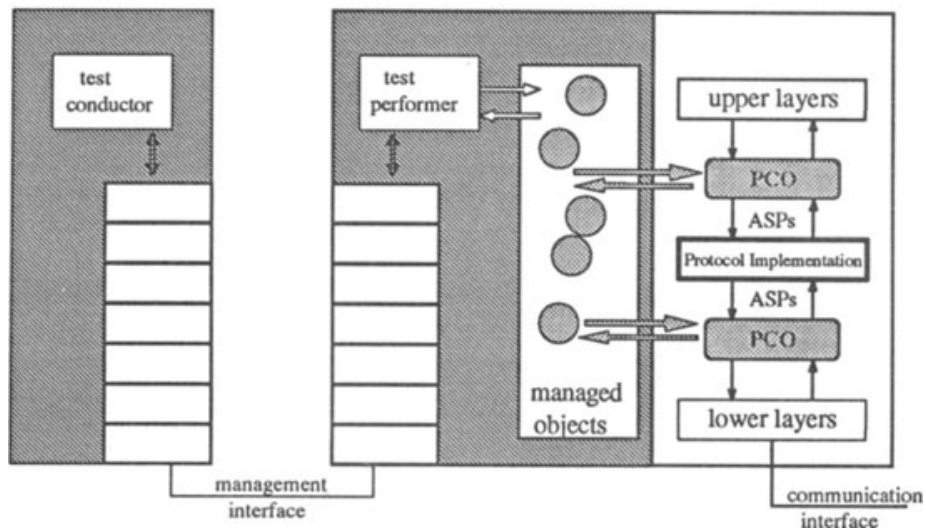

Figure 6. Protocol conformance testing using the Resource Boundary Test Category.

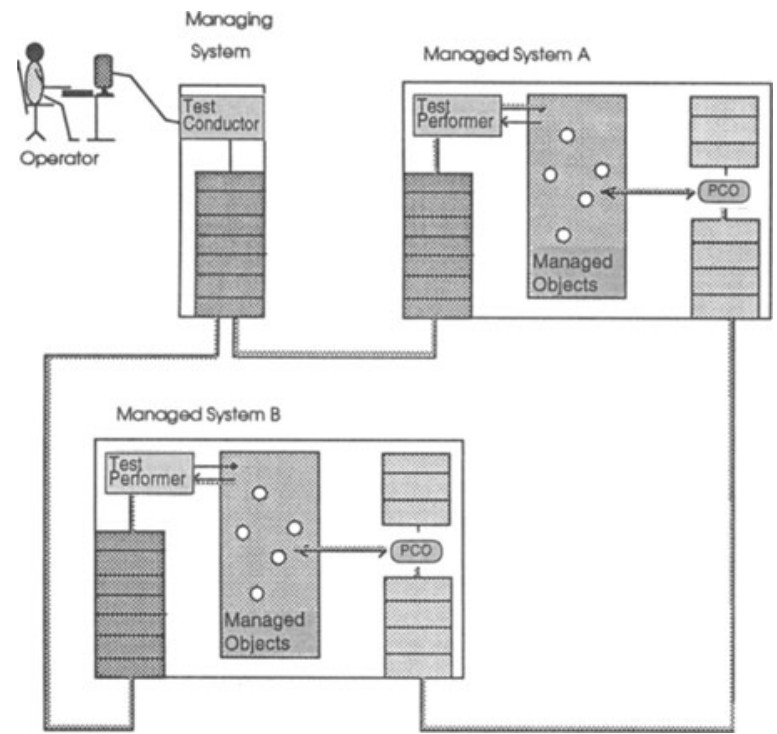

Figure 7: practical example of interoperability testing using OSI Management

\subsection{Costs and Benefits}

Several advantages and drawbacks have been identified of the newly described test technique. This section tries to quantify them and to indicate how disadvantages may become less important. 


\subsubsection{Costs}

To apply the OSI management test technique some modifications shall be made to the Protocol Management Models. These are documents that describe the management aspects of a specific protocol. The following additions shall be made:

- The protocol layers shall be modelled as managed objects

- The Service Access Points (SAPs) shall be modelled as PCOs.

- The Abstract Service Primitives (ASPs) and their parameters shall be explicitly listed in the information model.

These requirements are not very extensive in terms of paperwork, however they may cause some aversion regarding the explicit definition of ASPs. It should be noted that the definition of ASPs does not restrain their implementation.

It has been objected against these extensions that it still does impose implicit constraints on the implementation of internal interfaces. Such constraints are considered to be undesirable by manufacturers. A topic of further research would be the question how to overcome this objection.

Apart from the additional definitions extra functionality shall be implemented in the System Under Test. The following extensions are required:

- Implementation of PCOs. Experiences have shown [3] that the amount of code required for this is quite small, in comparison to the actual size of the protocol implementation. Furthermore this code is mainly generic, which means that it can be multiplied after being developed for one internal interface.

- Implementation of the Managed Objects and their behaviour, as specified in the relevant OSI management standards. This might imply indeed a substantial amount of code. However this is mainly code required for all kinds of management functions. The part for test management is relatively small.

\subsubsection{Benefits}

Application of the Resource Boundary Test Category will provide benefits in terms of both time and money. The following reductions in time can be achieved:

- Shorter development time of test suites. Test suites using the local test method do not need complex preambles to absorb the behaviour of the IUTs service user. They neither need to incorporate the declarations and values of the PDUs of the IUTs service user.

- Shorter test execution time. As the tests can concentrate on just the IUT aspects it is expected that traditional execution problems regarding the behaviour of other layers or application processes can be eliminated.

- Shorter time to market. Tests do not only take less time, they can also start earlier in the development process of the system under test. The system does not have to be complete prior to testing system parts.

In terms of money the following benefits can be achieved:

- Cheaper test systems. Instead of emulating the behaviour of the underlying protocol layers, the test systems have to support the OSI management procedures. This means that the same test system can be re-used for testing all kinds of implementations; just as long as the management interface is identical.

- Cheaper test development and execution. This is not only caused by the reduced time, but also because less specific knowledge is required for the development and execution of the tests. 
These considerations may become effective mainly after the introduction and acceptance of TMN and OSI management as the general management solution.

\section{CONCLUSIONS}

The difficulties associated with testing telecommunications software reach unacceptable levels due to increasing system complexity. Existing test methods are not powerful enough to test the systems efficiently. Therefore a new test technique has been developed, that provides direct access to the various system components to be tested. The test system will use OSI Management and the management interface to execute and control the tests within the System Under Test. The Resource Boundary Test provides the user with means to test parts of a system separately as if they were not encapsulated in the system. This type of test is very suitable for resources that have complex behaviour and cannot (completely) be tested by observing external communication interfaces.

With the application of the Resource Boundary Test Category it will be possible to test the protocols much cheaper and quicker. Especially the test coverage will be higher. However, this can only be achieved after full acceptation of its basic principles by designers and manufacturers of management systems.

\section{ACKNOWLEDGEMENTS}

We would like to thank Frank Bakker for reviewing and supporting our work during the last 2 years and, of course, Mischa Bart for his valuable contributions from the area of OSI management.

\section{REFERENCES}

[1] ISO/IEC 9646-1: July 1991, Information Technology, OSI Conformance Testing Methodology and Framework, Part 1: General Concepts.

[2] Zeng, H.X. and Rayner, D., The impact of the Ferry Concept on Protocol Testing. Protocol Specification, Testing, and Verification, V, North-holland publishers, 1986, pp. 519-531.

[3] Witteman, M.F. and Wuijtswinkel, R.C., ATM broadband testing using the ferry principle. Protocol Test Systems, VI, North-holland publishers, 1994, pp. 125-138.

[4] ISO/IEC, IS 7498-4 (=CCITT X.700); Information Processing Systems - Open Systems Interconnection - Basic Reference Model - Management Framework; 1989.

[5] ISO/IEC, IS 10040 (=CCITT X.701); Information Processing Systems - Open Systems Interconnection - Management Information Services - Systems Management Overview; 1991.

[6] ISO/IEC, IS 10065-1 (=CCITT X.720); Information Processing Systems - Open Systems Interconnection - Management Information Services - Structure of Management Information; Part 1: Management Information Model; 1991.

[7] ISO/IEC, IS 10164-12 (=CCITT X.745); Information Processing Systems - Open Systems Interconnection - Systems Management; Part 12: Test Management Function; October 1992.

[8] ISO/IEC, IS 10164-14 (=CCITT X.737); Information Processing Systems - Open Systems Interconnection - Systems Management; Part 14: Confidence and Diagnostic Test Categories; February 1992、 